\title{
Zur Ausschussbesetzung in Parlamenten: Berechnungsverfahren im Vergleich
}

\author{
Fred Hermsdorf
}

Zur Vorbereitung von Parlamentsbeschlüssen erfolgt eine Beratung in den entsprechenden Fachausschüssen des Parlamentes. Die Zuteilung der Ausschusssitze auf die Parlamentarier, das heißt genauer die Verteilung der Stimmenzahl im Ausschuss, ist entscheidend dafür, ob das Ausschussvotum im Parlament eine Mehrheit finden kann. Aus diesem Grund wird im Allgemeinen - und so auch im Deutschen Bundestag - versucht, die Ausschüsse als kleineres Spiegelbild des Parlamentes zu besetzen. Betrachtet man dagegen den Bundesrat, so ergibt sich ein anderes Bild. Hier erhält jedes Land im Ausschuss eine Stimme. Im Plenum aber schwankt die Zahl der Stimmen, die darüber hinaus einheitlich abgegeben werden müssen, je nach Einwohnerzahl des Landes zwischen drei und sechs. Daher bedeutet eine Mehrheit im Ausschuss nicht unbedingt eine Mehrheit im Plenum.

Bei Verhältniswahlsystemen liegt es nahe, Ausschüsse als kleineres Abbild des Parlamentes nach demselben Berechnungsverfahren zu besetzen, das auch für die Zusammensetzung des Parlaments herangezogen wird. Bei der Umsetzung eines Wahlergebnisses in Parlamentsmandate spielen aber oftmals neben dem Berechnungsverfahren die Wahlgesetze eine entscheidende Rolle. Überhangmandate, Ausgleichsmandate und regionale Untereinheiten wie Länder und Regierungsbezirke, in denen Ausgleiche vorgenommen werden, beeinflussen die Zusammensetzung eines Parlamentes ganz entscheidend. Damit die Ausschüsse das Parlament im Kleinen widerspiegeln, kann ihre Besetzung also nur auf der Basis der endgültigen Stärkeverhältnisse im Parlament erfolgen. Ein Rückgriff auf die prozentuale Verteilung der abgegebenen Stimmen der Wähler auf die verschiedenen Parteien ist kaum möglich.

Verwendet man die gängigen Berechnungsverfahren auch zur Ermittlung der Ausschussbesetzung, so ist Folgendes zu beachten: Diese Verfahren sind entwickelt worden unter der Forderung, dass alle Wähler mit der Stimme, die sie abgeben, den gleichen Einfluss auf das Wahlergebnis haben sollen. Ausschüsse dagegen sollen die Fraktionsstärken im Parlament widerspiegeln. Diese beiden Aussagen sind nicht ohne weiteres zur Deckung zu bringen. Bevor die Frage des Widerspiegelns genauer untersucht wird, sollen zwei Punkte angesprochen werden, die in den Zusammenhang der Problematik bei der Berechnung von Ausschussbesetzungen gehören, aber im Allgemeinen nicht als besonders erwähnenswert angesehen werden.

(1) Die Anzahl der Stimmen beziehungsweise Sitze muss ganzzahlig sein. Dass man einem Ausschuss nicht Bruchteile von Abgeordneten zuteilen kann, ist offensichtlich; für die Stimmenzahl ist dies aber prinzipiell denkbar. Solche eventuell zugeordneten Bruchstücke von Stimmen lassen sich aber nur schwer handhaben. Eine Bindung dieser Teilstimme an wen auch immer ist kaum möglich. Die Ganzzahligkeit ist somit die praktischere Variante.

Auf die sich dadurch ergebenden Folgen soll kurz hingewiesen werden: Da bei der Besetzung eines Ausschusses im Allgemeinen die exakte Proportionalität (Ausschussgröße * Größe der Fraktion / Gesamtgröße des Parlaments) nicht eingehalten werden kann, muss auf- oder abgerundet werden. Der so gewonnene beziehungsweise verlorene Anteil wächst 
umso mehr, je kleiner die Ausschussgröße festgelegt wird. Außerdem wirken sich die durch Rundung erfolgten Abweichungen umso stärker aus, je kleiner die Fraktion ist. Hat zum Beispiel eine Fraktion eine exakte Proportionalität von 1,5 in einem Ausschuss von sechs Mitgliedern, erhält aber nur einen Sitz, dann besitzt sie nur ein Sechstel statt ein Viertel der Stimmen, die ihr unter Proportionalitätsgesichtspunkten eigentlich zustehen. Sie kommt also nur auf circa 67 Prozent ihres eigentlichen Gewichtes. Hat aber eine Fraktion eine exakte Proportionalität von 4,5 wieder bei einer Ausschussgröße von sechs, erhält aber nur vier Stimmen, dann besitzt sie zwei Drittel statt drei Viertel der Stimmen. Sie kommt also schon auf circa 89 Prozent ihres eigentlichen Gewichtes. In beiden Fällen wurde jeweils von 0,5 abgerundet. Die Vermutung liegt also nahe, dass die Einhaltung der Ganzzahligkeit der Stimmen die Ursache mannigfaltiger Probleme ist.

(2) Die Festlegung auf eine bestimmte Ausschussgröße ist in den meisten Fällen nicht zwingend, sondern unterliegt dem Willen des Parlamentes.

\section{Was bedeutet "Widerspiegelung“?}

Mit zwei Kriterien kann überprüft werden, was „widerspiegeln“ bedeuten soll. Ohne derartige Kriterien für die zahlenmäßige Beziehung Parlament / Ausschuss wird der Begriff vermutlich je nach Erwartung anders interpretiert und als unschön empfundene Eigenschaften von Berechnungsverfahren (zum Beispiel die so genannten unlogischen Sprünge bei Hare / Niemeyer) könnten besondere Bedeutung erlangen, obwohl sie auf die Zusammensetzung bei einer festgelegten Ausschussgröße keinen Einfluss haben.

(1) Eine allgemein genannte und auch nahe liegende Forderung lautet: Die Verteilung der Sitze im Ausschuss auf die Fraktionen soll proportional zu dem Anteil ihrer Sitze im Parlament sein. Dieses scheinbar offensichtliche Verlangen stellt sich bei genauer Betrachtung als gar nicht so einfach zu handhaben dar. Bei der Vorstellung von Proportionalität ist sicher auch gemeint, dass das Größenverhältnis der einzelnen Fraktionen zueinander abgebildet wird. Betrachten wir zwei Beispiele: Im Nordrhein-Westfälischen Landtag sind gegenwärtig die CDU mit 89, die SPD mit 74, die Grünen mit zwölf und die FDP ebenfalls mit zwölf Sitzen vertreten. Erhalten in einem Ausschuss Grüne oder FDP mindestens einen Sitz, dann müssen auf die SPD mindestens sechs und auf die CDU mindestens sieben Sitze entfallen. Damit kann man eine Einhaltung der Größenrelation erst ab einer Ausschussgröße von mindestens $1+1+6+7=15$ erwarten. Macht man dieselbe Rechnung für den Deutschen Bundestag auf, so ergibt sich bei der Sitzverteilung in der 16. Wahlperiode von CDU/CSU: 226, SPD: 222, FDP: 61, Linke: 54 und Grüne: 51 Sitze folgendes Bild: Erhalten in einem Ausschuss FDP, Linke und Grüne mindestens einen Sitz, dann müssen der SPD mindestens vier (oder fünf) und der CDU/CSU mindestens vier (oder fünf) Sitze zugeteilt werden. Somit ergibt sich eine Mindestgröße für einen Ausschuss von elf (oder 13) Sitzen.

Nur bestimmte Ausschussgrößen können also die Einhaltung dieser Verhältnisrelationen gewährleisten. Auch wird deutlich, dass weder die Größe des Parlamentes noch die Zahl der Fraktionen einen Rückschluss auf die möglichen Ausschussgrößen erlaubt. Diese Überlegungen zeigen, dass die Forderung nach dieser Form der Proportionalität wenig geeignet ist, da diese nur bestimmte Ausschussgrößen zulässt. 
Unter Einhaltung der Proportionalität wird auch verstanden, dass das Ergebnis der Berechnung innerhalb der Quote liegt. Damit ist Folgendes gemeint: (Stimmenzahl der Fraktion) * Ausschussgröße / (Gesamtstimmenzahl im Parlament) ergibt eine ganze Zahl g größer gleich 0 und einen Rest $r$ größer gleich 0 und kleiner 1 . Eine Berechnungsmethode liefert ein Ergebnis innerhalb der Quote, wenn sie den Wert g oder g+1 ergibt. Am Beispiel des Bundestages: Die Gesamtstimmenzahl im Parlament ist 614. Die geforderte Ausschussgröße sei 15. Für die CDU/CSU ergibt sich $\left(15^{*} 226\right) / 614=5,52$. Damit ist g = 5. Für diese Fraktion liegt das Ergebnis innerhalb der Quote, wenn ihr durch das Berechnungsverfahren fünf oder sechs Sitze in einem Ausschuss von 15 Mitgliedern zugeteilt werden.

Zur Einhaltung der Proportionalität wird somit als erstes Kriterium gefordert: Die Ausschussbesetzung soll bei Ableitung aus den Fraktionsstärken im Parlament innerhalb der Quote liegen.

(2) Das zweite Kriterium zur Bestimmung von „Widerspiegelung“ bezieht sich auf die „Machtverhältnisse“ im Parlament. Danach soll die Mehrheit im Ausschuss der Mehrheit im Parlament entsprechen, wobei nicht nur die gegenwärtige, sondern auch politisch denkbare Mehrheiten gemeint sind. Dieses Kriterium ist schon deswegen besonders wichtig, weil bei Koalitionsregierungen auch ohne Neuwahl niemals eine Änderung der Regierungskonstellation auszuschließen ist. In einem solchen Fall wirkt es befremdlich, wenn entweder die neue Regierung in den Ausschüssen keine Mehrheit hat oder ein Wechsel der Berechnungsmethode erforderlich wird, um für die neue Regierung die Mehrheit in den Ausschüssen zu sichern, oder sogar neue zusätzliche Vorschriften aufgestellt werden müssen und dies alles, ohne dass sich die Zusammensetzung des Parlamentes verändert hat.

Leider kann man aber nicht sicherstellen, dass bei jeder Ausschussgröße überhaupt eine Besetzung ermittelt werden kann, die alle im Parlament möglichen Mehrheiten reproduziert. Dies sei am Beispiel eines Parlamentes von drei Fraktionen ohne absolute Mehrheit einer Fraktion gezeigt: In diesem Fall bilden alle möglichen Koalitionen von zwei Fraktionen eine Mehrheit im Parlament. Soll ein Ausschuss mit vier Mitgliedern besetzt werden, muss eine Fraktion - unabhängig von der Berechnungsmethode - mindestens zwei Sitze erhalten. Somit besitzt sie im Ausschuss mindestens die Hälfte der Sitze unabhängig davon, ob sie der Regierung angehört oder nicht. Eine Mehrheit der beiden anderen Fraktionen in diesem Ausschuss ist also niemals möglich, obwohl eine entsprechende Koalition im Gesamtparlament die Mehrheit besitzt. Im selben Parlament stellen dagegen Ausschussgrößen ab fünf für die Mehrheitserhaltung kein Problem dar.

Die Forderung, die Ausschussbesetzung solle die Parlamentsbesetzung widerspiegeln, ist also einleuchtend, aber nicht in jedem Fall umsetzbar.

\section{Was leisten gängige Verfahren?}

Die mathematische Präzisierung des Begriffs „Widerspiegelung“ hilft, gängige Verfahren zur Ausschussbesetzung auf ihre Tauglichkeit zu überprüfen. Dabei wird gegebenenfalls durch die Angabe eines Gegenbeispiels gezeigt, dass oftmals erwartete Eigenschaften nicht vorhanden sind. 


\subsection{Verfahren Hare / Niemeyer}

Da dieses Verfahren immer von der exakten Proportionalität ausgeht und die Zuteilung der restlichen Sitze anhand der größten Reste vornimmt, ist die Quote (Kriterium 1) stets eingehalten. Da aber Hare / Niemeyer für jede beliebige Ausschussgröße eine Zusammensetzung ermittelt, ist die Einhaltung von Kriterium 2 nicht immer gewährleistet (wie oben gezeigt existieren Konstellationen, für die es keine Lösung unter Einhaltung von Kriterium 2 gibt). Leider ermittelt dieses Verfahren aber auch dann nicht immer die Lösung zur Einhaltung von Kriterium 1 und 2, wenn sie existiert.

Zur Erläuterung wird eine geringfügig abgewandelte Verteilung der oben angegebenen Zusammensetzung des Nordrhein-Westfälischen Landtages als Ausgangspunkt genommen. Durch diese Modifikation werden die Mehrheitsverhältnisse im Landtag nicht verändert. Sie hat hier allein den Grund, nicht durch eine Diskussion über Losverfahren bei Gleichheit von Fraktionsstärken von der eigentlichen Aussage abzulenken. Im Landtag selbst, der das Verfahren von Hare / Niemeyer anwendet, wird das Losverfahren zwischen Grünen und FDP zurzeit dadurch umgangen, dass in solchen Fällen die Entscheidung immer zugunsten der Grünen ausfällt, da sie bei der Landtagswahl mehr Stimmen als die FDP auf sich vereinigen konnten.

Läge etwa folgende Zusammensetzung vor: CDU 89, SPD 74, Grüne 13 und FDP 12 Sitze, verfügten CDU und FDP mit 101 von 188 Stimmen über die Mehrheit. Als exakte Proportionalitäten ergeben sich bei einem Ausschuss von sieben Mitgliedern: CDU: 3,31; SPD: 2,76; Grüne: 0,48 und FDP: 0,45. Nach Hare / Niemeyer errechnet sich folgende Ausschussbesetzung: CDU: 3, SPD: 3, Grüne: 1 und FDP kein Sitz. CDU und FDP besitzen danach bei dieser Ausschussgröße keine Mehrheit. Eine Verteilung: CDU: 3, SPD: 2, Grüne: 1 und FDP: 1 Sitz dagegen erfüllt sowohl Kriterium 1 als auch Kriterium 2.

\subsection{Verfahren St. Laguë/ Schepers}

Dieses Verfahren wird vom Bundestag zur Ermittlung seiner Ausschussbesetzungen angewendet. Bei einer Ausschussgröße von 15 Mitgliedern ergeben sich mit den oben aufgeführten Fraktionsstärken für die CDU/CSU 6, die SPD 5, die FDP 2, die Linke 1 und die Grünen ebenfalls 1 Sitz. Wäre die Regierung aus SPD, Linke und Grünen gebildet worden, was mit 327 von 614 Abgeordneten rechnerisch möglich gewesen wäre, hätte diese Regierungskoalition mit sieben von 15 Mitgliedern in diesem Ausschuss keine Mehrheit gehabt. Die sichere Einhaltung von Kriterium 2 kann hier aber ebenso wie bei Hare / Niemeyer (Berechnung für jede Ausschussgröße möglich) nicht erwartet werden.

Betrachtet man die exakte Proportionalität, so ergeben sich in diesem Fall: CDU/CSU: 5,52; SPD: 5,42; FDP: 1,49; Linke: 1,32 und Grüne: 1,25. Mit der Verteilung CDU/CSU 6, SPD 6, FDP, Linke und Grüne je ein Sitz wären auch hier Kriterium 1 und Kriterium 2 erfüllt gewesen.

Dass St. Laguë / Schepers nicht nur bei der Einhaltung von Kriterium 2 versagen kann, sondern auch die Einhaltung von Kriterium 1 nicht sicherstellt, soll durch folgendes Beispiel eines fiktiven Parlamentes mit den Parteien A, B, C, D und E demonstriert werden: Bei einer Sitzverteilung A: 236, B: 235, C: 49, D: 48 und E: 48 betrüge die exakte Propor- 
tionalität bei 21 Mitgliedern im Ausschuss: A: 8,05; B: 8,01; C: 1,67; D: 1,64 und E: 1,64. Das Verfahren St. Laguë/ Schepers ergibt aber A: 8; B: 7(!); C: 2; D: 2 und E: 2.

\subsection{Verfahren $d$ Hondt}

Die Aussagen zum Verfahren von St. Laguë / Schepers gelten hier entsprechend und werden daher nicht wiederholt. Ein Beispiel verdeutlicht die Problematik dieser Berechnungsmethode: In einem Bundestagsausschuss (Zusammensetzung des Bundestages s.o.) mit 19 Mitgliedern verteilen sich die Sitze nach $d$ Hondt wie folgt: CDU/CSU 8, SPD 7, FDP 2, Linke 1 und Grüne 1. SPD, Linke und Grüne stellen zusammen nur neun Mitglieder und damit nicht die Mehrheit.

Die exakten Proportionalitäten lauten: CDU/CSU: 6,99; SPD: 6,87; FDP: 1,89; Linke: 1,67 und Grüne: 1,58. Die Zahl der CDU/CSU zeigt sofort, dass hier auch Kriterium 1 verletzt ist. Die Verteilung CDU/CSU: 7, SPD: 7, FDP: 2, Linke: 2 und Grüne: 1 Sitz erfüllt dagegen sowohl Kriterium 1 als auch Kriterium 2.

\subsection{Verfahren der Mehrheitstreue}

Hierbei wird zuerst die Einhaltung von Kriterium 2 und falls möglich die Einhaltung von Kriterium 1 sichergestellt. Gibt es für eine bestimmte Ausschussgröße keine Verteilung, die Kriterium 2 erfüllt, wird dies angezeigt. Kann Kriterium 2, jedoch nicht Kriterium 1 erfüllt werden, so wird der Bereich der Quote vergrößert. Dies erfolgt in der Art, dass die kleinste Abweichung von der exakten Quote ermittelt wird. Hier wird anders als bei den obigen Methoden nicht das Rechenverfahren, sondern die Erfüllung der Kriterien in den Vordergrund gestellt. ${ }^{1}$

Ausgehend von der oben angegebenen Zusammensetzung des Nordrhein-Westfälischen Landtags soll als Beispiel ein Ausschuss mit zwölf Mitglieder besetzt werden. Aus der Sitzverteilung im Parlament ergeben sich folgende mögliche Mehrheiten: CDU mit SPD, CDU mit Grünen, CDU mit FDP, SPD mit Grünen und FDP.

Exakte Proportionalitäten

\begin{tabular}{|c|c|c|c|}
\hline \multicolumn{4}{|l|}{$\begin{array}{l}\text { CDU: 5,71 } \\
\text { Hare / Niemeyer }\end{array}$} \\
\hline CDU: 5 & SPD: 5 & Grüne: 1 & FDP: 1 \\
\hline \multicolumn{4}{|c|}{ St. Laguë / Schepers } \\
\hline $\begin{array}{l}\text { CDU: } 5 \\
d \text { Hondt }\end{array}$ & SPD: 5 & Grüne: 1 & FDP: 1 \\
\hline $\begin{array}{l}\text { CDU: } 7 \\
\text { Mehrheitstreue }\end{array}$ & SPD: 5 & Grüne: 0 & FDP: 0 \\
\hline CDU: 5 & SPD: 3 & Grüne: 2 & FDP: 2 \\
\hline
\end{tabular}

1 Ein Verfahren zur Berechnung der Sitzverteilungen findet sich in: Fred Hermsdorf, Mehrheitstreue und Proportionalität: Zur Berechnung von Sitzverteilungen in Parlamenten, in: ZParl, 27. Jg. (1996), H. 1, S. 5 ff. 
Während die ersten drei Verfahren Kriterium 2 verletzen (zum Beispiel keine Mehrheit für CDU und FDP beziehungsweise für SPD, Grüne und FDP), gelingt bei der Methode der Mehrheitstreue die Einhaltung von Kriterium 2 nur durch Verletzung von Kriterium 1, wie man unmittelbar durch Vergleich mit den exakten Proportionalitäten sieht. Mit den weiter oben angegebenen Bezeichnungen liegt jetzt die Verteilung nicht in dem Bereich $\mathrm{g}$ und $\mathrm{g}+1$, sondern $\mathrm{g}-1$ (beziehungsweise 0 , falls g-1 negativ ist) und $\mathrm{g}+2$. Zur Verdeutlichung wird dies in Tabelle 1 noch einmal zusammengefasst.

\begin{tabular}{|l|c|c|}
\hline Tabelle 1: & $\mathrm{g}$ & zugeteilte Stimmenzahl \\
\hline Partei & 5 & 5 \\
\hline CDU & 4 & 3 \\
\hline SPD & 0 & 2 \\
\hline Grüne & 0 & 2 \\
\hline FDP & & \\
\hline
\end{tabular}

\title{
3. Die richtige Wahl: Verfahren der Mehrheitstreue und Hare / Niemeyer
}

Die Verfahren von St. Laguë / Schepers und d Hondt sind unter der hier erfolgten Definition von Widerspiegelung nicht geeignet, um Ausschussbesetzungen zu berechnen, da sie weder die Einhaltung von Kriterium 1 noch von Kriterium 2 sicherstellen können, also weder liegt die Ausschussbesetzung innerhalb der von den Fraktionsstärken abgeleiteten Quote, noch entsprechen die Mehrheiten im Ausschuss allen möglichen Mehrheitsvarianten im Parlament. Legt man als oberstes Ziel die Einhaltung von Kriterium 1 fest, ist das Verfahren von Hare / Niemeyer die richtige Wahl. Wünscht man dagegen - wenn immer überhaupt möglich - die Einhaltung von Kriterium 2, muss man zum Verfahren der Mehrheitstreue greifen.

\section{„Hammelsprung“ und Parlamentssymbolik im Reichstagsgebäude der Kaiserzeit. Ergänzungen zum Beitrag von Kai Zäble in Heft 2/2007 der ZParl*}

\author{
Michael F. Feldkamp
}

Der langjährige Parlamentspräsident des Deutschen Reichstages während der Weimarer Zeit, Paul Löbe (SPD), hatte als Mitglied des 1. Deutschen Bundestages, befragt nach der Herkunft des Begriffes „Hammelsprung“, sich nur vage an ein Intarsienbild über einer der Abstimmungstüren im Reichstagsgebäude erinnert, durch das die Abgeordneten gingen,

* Für die Unterstützung bei den Recherchen danke ich herzlich Barbara Wieners-Horst, Redaktions-
stab der Gesellschaft für deutsche Sprache beim Deutschen Bundestag. 